\title{
Multiparametric dependence of hydrogen Stark profiles asymmetry
}

\author{
A.V. Demura ${ }^{1, a}$, G.V. Demchenko ${ }^{1}$, and D. Nikolić ${ }^{2, b}$ \\ 1 IVEPT, RRC "Kurchatov institute", Kurchatov Square 1, 123182 Moscow, Russia \\ 2 Department of Physics, Western Michigan University, Kalamazoo MI 49008, USA \\ Received 17 October 2007 \\ Published online 14 November 2007 - (C) EDP Sciences, Società Italiana di Fisica, Springer-Verlag 2007
}

\begin{abstract}
This addendum states more accurately our understanding of the foundations of Advanced Generalized Theory (AGT) and corrects its bibliographic entry. To avoid any further misunderstandings readers are encouraged to consult the original work published by the AGT authors.
\end{abstract}

PACS. 32.70.Jz Line shapes, widths, and shifts - 32.60.+i Zeeman and Stark effects - 52.20.Fs Electron collisions - 52.20.Hv Atomic, molecular, ion, and heavy-particle collisions - 52.25.Os Emission, absorption, and scattering of electromagnetic radiation

1. Bibliography item [67] should be substituted with: E. Oks, J. Phys. B 35, 2251 (2002)

2. The text below should be substituted for the third, fourth, and fifth paragraphs of Section 6.6.

The GT and AGT [66] employ atomic states dynamically dressed by the projection of the electron microfield on the ion microfield (and by the ion microfield itself). The parallel and perpendicular projections of the electron microfield are considered separately: the parallel contribution - by using adiabatic non-perturbative approach, and the perpendicular contribution - by using the perturbation expansion up to the second order (the latter being used in ST as well $[48,49,56])$. Such a separation is in fact common in the theory of magnetic resonance, laser physics etc., where it is based on the general notions of slow and rapid perturbations. However, in magnetic resonance the parallel and perpendicular magnetic fields often have different physical origins, while in GT and AGT both components originate from the same electron microfield.

This approach alone invokes dramatic changes in the important theoretical predictions [66]. For example, the so-called broadening widths and shift functions $A(z)$ and $B(z)$ (see $[24,48,49]$ ) in AGT become redefined and parametrically dependent (quite strongly) on the ion microfield, and these start to oscillate as a function of the impact parameter as opposed to the monotonic behavior of their analogs in ST $[24,56]$. In addition, in Figure 4.1 in [66] one can find negative values of $A_{-}^{\mathrm{AGT}}(z)$ for small values of impact parameter. This result of AGT might seem unusual, but not alarming: the width of the line is controlled in a complicated way by three different "width functions" $A_{-}^{\mathrm{AGT}}(z), A_{+}^{\mathrm{AGT}}(z)$ and $A_{x}^{\mathrm{AGT}}(z)$ (see $[66]$ ), rather than by $A_{-}^{\mathrm{AGT}}(z)$ alone. The total electronic collision shift also becomes more complex, being related to the sum of contributions considered by Griem and several contributions derived in the framework of GT and AGT [66,67].

The appearance of adiabatic terms in the impact theory should be welcomed, since their absence was a longstanding methodological drawback in the derivation of the impact approximation. However, AGT should also conform to some boundaries that have not yet been established.

\footnotetext{
a e-mail: Alexander.Demura@hepti.kiae.ru

b e-mail: dragan.nikolic@wmich.edu
} 